\title{
CORRELATION OF MICROMETRIC PARAMETERS WITH QUALITY OF OOCYTES IN
} NILI-RAVI BUFFALOES

\author{
M. Nawaz, M. Saleem, M.U. Iqbal and A. Riaz \\ Department of Theriogenology, Faculty of Veterinary Science, University of Veterinary and Animal Sciences, Lahore \\ 54000, Pakistan \\ *Corresponding author's E-mail: dramjadriaz@uvas.edu.pk \\ The paper was presented in International Buffalo Congress 2019, February 18-20, Lahore, Pakistan
}

https://doi.org/10.36899/JAPS.2020.1.0028

Published online January 02, 2020

\begin{abstract}
Detailed visibility of ooplasm plays a pivotal role in advanced reproductive bio techniques (ICSI, SCNT). To improve visibility, oocytes are usually handled after cumulus denuding. This study was conducted to establish buffalo oocyte quality estimation in denuded form. For this purpose, correlations were recognized among the size of oocyte, ooplasm and zona pellucida (ZP) with oocyte quality in Nili-Ravi buffalo. From slaughter house based ovaries, oocytes $(\mathrm{n}=150)$ were collected by aspiration of follicles and categorized into A, B, C, and D grades based on the number of layers of cumulus cell. After denuding with hyaluronidase enzyme, the detailed micrometric study was performed under inverted microscope at $40 \mathrm{X}$ by using micro imaging software (cellSens Dimension 1.16). The data were analyzed by correlation test using a statistical analysis system (SAS 9.1). The results demonstrated as the followings, the mean oocytes diameter $(149.76 \pm 0.99 \mu \mathrm{m})$, the width of zona pellucida $(12.84 \pm 0.23 \mu \mathrm{m})$, ooplasm diameter $(117.5 \pm 0.70 \mu \mathrm{m})$, oocyte area $\left(17804 \pm 136 \mu \mathrm{m}^{2}\right)$ and ooplasm area $\left(11308.09 \pm 75 \mu \mathrm{m}^{2}\right)$. There was a strong positive correlation between the ooplasm diameter and oocytes diameter in $\mathrm{A}$ and $\mathrm{B}$ grade oocytes $(\mathrm{r}=0.7792)$ as compared to $\mathrm{C}$ and $\mathrm{D}$ grade oocytes $(\mathrm{r}=0.5967)$. However, there was moderate positive correlation between the width of zona pellucida and oocytes diameter in $\mathrm{C}$ and $\mathrm{D}$ grade oocytes $(\mathrm{r}=0.6530)$ as compared to the $\mathrm{A}$ and $\mathrm{B}$ grade oocytes $(\mathrm{r}=0.5287)$. In conclusion, the greater the ooplasm diameter and lesser the width of zona pellucida give rise to the good quality oocytes. Therefore, it is implied that for better developmental competence, the oocytes with bigger ooplasm and thinner zona pellucida should be processed.
\end{abstract}

Key words: Nili-Ravi Buffalo oocyte, micrometry, correlation.

\section{INTRODUCTION}

Assisted reproductive technologies have revolutionized the dairy industry in the past century for the rapid multiplication and up gradation of superior genetics [Moore et al., 2017]. Intracytoplasmic sperm injection (ICSI) is already a well established technique in human for infertile couples while in livestock somatic cell nuclear transfer (SCNT) and transgenesis are emerging biotechnologies. Usually, reproductive biotechniques involve basic steps like oocyte collection, grading, in-vitro maturation, sperm capacitation, in-vitro fertilization and in-vitro culture. For advanced reproductive bio-techniques, the success rates are reported to be influenced by several factors which include width of zona pellucida [Bertrand et al., 1996], oocyte diameter [Yousaf et al., 2003], and correlation of width of zona pellucida with clinical pregnancy rate [Gabrielsen et al., 2000].

Previous studies depicted that oocyte diameter influenced maturation rate in buffalo [Yousaf et al., 2003]. The thickness of zona pellucida is also reported to influence negatively fertility rate [Hatif et al., 2016]. Further, in humans the variation in the thickness of zona pellucida is also reported to have a strong correlation with clinical pregnancy rate during ICSI [Gabrielsen et al., 2000].

During micromanipulation, the oocytes are usually handled in the denuded form to improve visibility. However, very limited information is available to evaluate the quality of oocytes in denuded form. Usually in bovine, oocyte grading protocols are based on the cumulus layer numbers surrounding oocyte [Kastrop et al., 1990]. Only a few studies are available for the micrometric evaluation of bovine oocytes [Fair et al., 1995; Otoi et al., 1997] but such information is still lacking in the Nili-Ravi buffalo. The objective of the present study was to establish oocyte quality correlation by its micrometric evaluation.

\section{MATERIALS AND METHODS}

This study was conducted during 2018 in the embryology laboratory of University of Veterinary and 
Animal Sciences, Ravi campus, Pattoki. The experiments were conducted using the reagents of Sigma Chemical Company (St. Louis, MO, USA) and Gibco (China).

Collection of ovaries: Buffalo ovaries were collected from a local slaughter house (Nili-Ravi: 8-12 years of age having mixed parity) with clinically normal reproductive tracts. The ovaries were carried to the laboratory within 2 hours of slaughtering at $30-35^{\circ} \mathrm{C}$ in a thermo flask having $0.9 \%$ normal saline solution containing sodium chloride (Sigma; cat \# S5886), and 100000 IU/L penicillin (Sigma; cat \# P3032). After arriving in the laboratory, the ovaries were washed in normal saline for thrice to remove the contamination.

Aspiration of the cumulus oocyte complexes: The cumulus oocyte complexes (COC's) were harvested by aspirating the small $(<6 \mathrm{~mm})$ and medium $(6-10 \mathrm{~mm})$ size follicles [Vieira et al., 2014] with the help of 18 gauge needle attached to a $10 \mathrm{ml}$ syringe containing our prepared washing media as an oocyte recovery media. After searching of the COC's, the oocytes were washed for three times in our prepared washing media, pre warmed at $38.5^{\circ} \mathrm{C}$ for 30 minutes, containing TCM 199 (Gibco; cat \# 31100-035), Hepes (acid)(Sigma; cat \# H6147), Hepes (salt) (Sigma; cat \# H3784), EDTA Na (Sigma; cat \# E6511), Pyruvate (Sigma; cat \# P4562), Penicillin(Sigma; cat \# P3032),Streptomycin (Sigma; cat \# S1277), FBS (Gibco), and Heparin sodium (Sigma; cat \# H3393).

Grading of the oocytes: The COC's were categorized into $\mathrm{A}, \mathrm{B}, \mathrm{C}$, and $\mathrm{D}$ grades based on the number of layers of cumulus cells and integrity of ooplasm. COC's with more than 5 cumulus layers, homogenous cytoplasmic appearance and unexpanded cumulus mass were categorized into A grade while COC's with up to three cumulus layers, homogenous cytoplasmic appearance and unexpanded cumulus mass were grouped into B grade COCs. C grade COCs were characterized by irregular and dark ooplasm with partially denuded or less than three cumulus layers. Denuded oocytes with inhomogeneous cytoplasm were classified into D grade oocytes [Neglia et al., 2003].

Denuding of oocytes: The COC's were denuded completely by pipetting of oocytes in the washing media containing $0.3 \mathrm{mg} / \mathrm{ml}$ hyaluronidase (Sigma).

Micrometry of the oocytes: Micrometric studies (Oocyte diameter, ooplasam diameter, zona pellucida width, ooplasm area and oocyte area) of Nili-Ravi buffalo oocytes were performed under Inverted microscope (IX73, Olympus) at $40 \mathrm{X}$ by using micro imaging software (cellSens Dimension 1.16). All the measurements were repeated for thrice at different angles, to reduce the error, in all the four categories $(\mathrm{A}, \mathrm{B}, \mathrm{C}$ and $\mathrm{D}$ grade) of oocytes.

Statistical analysis: The correlation between oocyte diameter, ooplasm diameter and width of zona pellucida was tested using correlation test. The partial correlation was estimated between two variables by eliminating the effect of third variable. The variables were considered weak, moderate and strongly correlated when the range of correlation coefficient was between $(0.30-0.50,0.50$ 0.70, and 0.70-0.90) respectively [Mukaka et al., 2012]. All the data were analyzed using Statistical Analysis System (SAS Institute, Inc., Cary, NC, USA).

\section{RESULTS}

The mean values of different micrometric parameters (oocytes diameter, width of zona pellucida, ooplasm diameter, and oocyte area and ooplasm area) of Nili-Ravi buffalo oocytes has been presented in the Table 1.The results demonstrated the mean oocytes diameter was $(149.76 \pm 0.99 \mu \mathrm{m})$, the width of zona pellucida $(12.84 \pm 0.23 \mu \mathrm{m})$, ooplasm diameter $(117.5 \pm 0.70 \mu \mathrm{m})$, oocyte area $\left(17804 \pm 136 \mu \mathrm{m}^{2}\right)$ and ooplasm area was $\left(11308.09 \pm 75 \mu \mathrm{m}^{2}\right)$ in Nili-Ravi buffalo. The correlation of oocytes quality with their micrometric evaluation (correlation of oocytes diameter with ooplasm diameter and correlation of oocyte diameter with width of zona pellucida) has been depicted in Fig. 1. There was a strong correlation between oocyte diameter and ooplasm diameter in $\mathrm{A}+\mathrm{B}$ grade oocytes as compared to $\mathrm{C}+\mathrm{D}$ grade oocytes $(\mathrm{r}=0.7792$ vs $\mathrm{r}=0.5967)$ respectively. However, there was a moderate correlation between oocyte diameter and width of zona pellucida in $\mathrm{C}+\mathrm{D}$ grade oocytes as compared to $\mathrm{A}+\mathrm{B}$ grade oocytes $(\mathrm{r}=0.6530$ vs $\mathrm{r}=0.5287)$ respectively.

Table 1. Micrometric evaluation of oocytes in NiliRavi buffalo.

\begin{tabular}{llc}
\hline Variables & Units & $\begin{array}{c}\text { Mean Value } \\
(\mathbf{n = 1 5 0 )}\end{array}$ \\
\hline Oocyte diameter & $\mu \mathrm{m}$ & $149.76 \pm 0.99$ \\
Ooplasm diameter & $\mu \mathrm{m}$ & $117.50 \pm 0.70$ \\
Zona pellucida width & $\mu \mathrm{m}$ & $12.84 \pm 0.23$ \\
Oocyte area & $\mu \mathrm{m}^{2}$ & $17804 \pm 136$ \\
Ooplasm area & $\mu \mathrm{m}^{2}$ & $11112.3 \pm 75$ \\
\hline
\end{tabular}

All the measurements were taken under the inverted microscope (Olympus IX73) attached with a DP74 camera (400X) using micro imaging software (cellSens Dimension 1.16). 


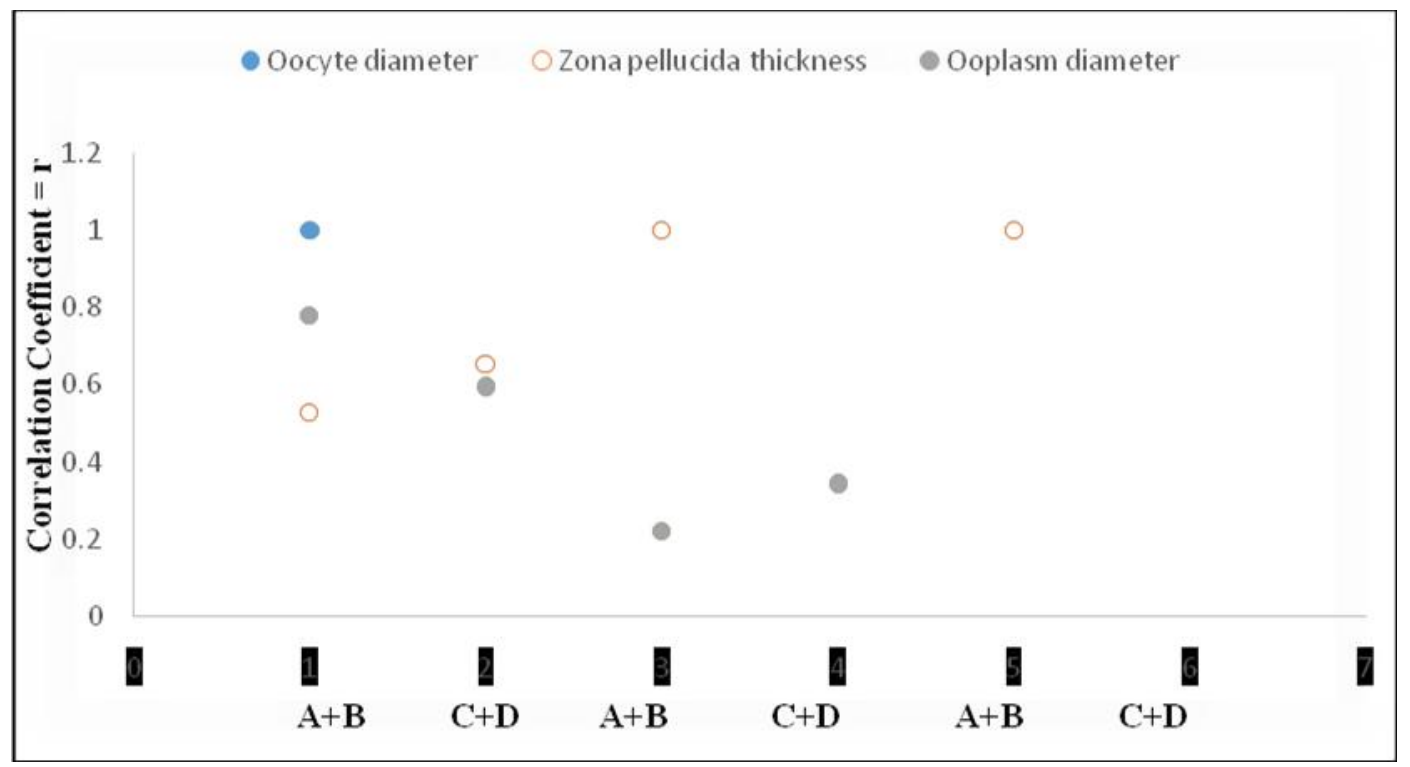

Fig. 1. Correlation of oocyte diameter, with zona pellucida thickness and ooplasm diameter in relation to quality of oocytes in Nili-Ravi buffalo.

\begin{tabular}{|c|c|c|c|c|c|}
\hline & \multicolumn{2}{|c|}{ Oocyte Diameter } & \multicolumn{2}{|c|}{ Zona pellucida thickness } & Ooplasm diameter \\
\hline & $\mathrm{A}+\mathrm{B}$ & $\mathrm{C}+\mathrm{D}$ & $\mathrm{A}+\mathrm{B}$ & $\mathrm{C}+\mathrm{D}$ & $\mathrm{A}+\mathrm{B} \quad \mathrm{C}+\mathrm{D}$ \\
\hline Oocyte diameter & & & & & \\
\hline Zona pellucida thickness & 0.528 & 0.653 & & & \\
\hline Ooplasm diameter & 0.779 & 0.596 & 0.221 & 0.344 & 1 \\
\hline
\end{tabular}

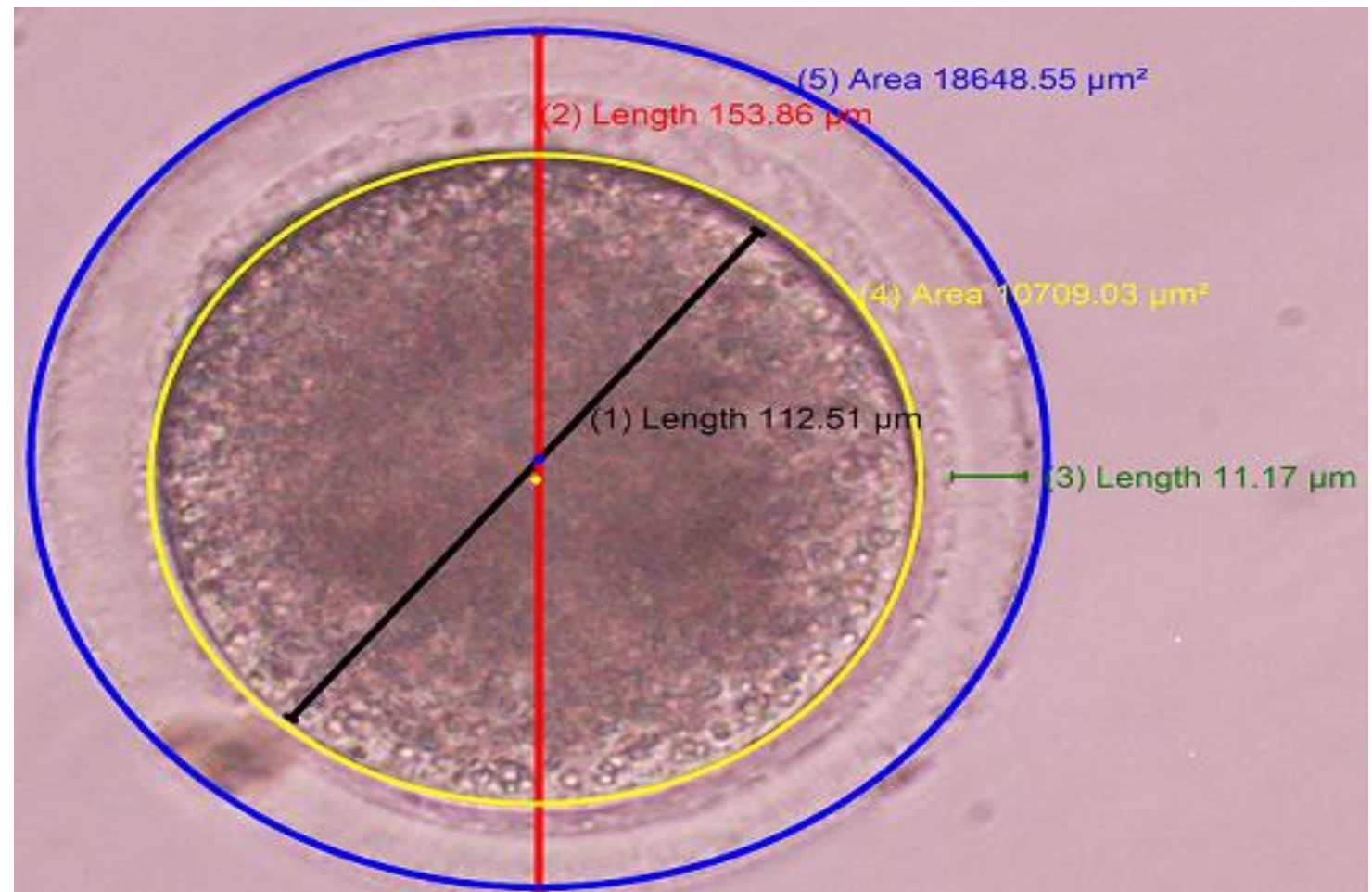

Fig. 2. Represents the ooplasm diameter (length 1), oocyte diameter (length 2), zona pellucida thickness (length 3), ooplasm area (length 4), oocyte area (length 5) in Nili-Ravi buffalo. All the measurements were taken under the inverted microscope (Olympus IX73) attached with a DP74 camera (400X) using micro imaging software (cellSens Dimension 1.16). 


\section{DISCUSSION}

To best of author's knowledge, this is the first study, conducting the micrometric evaluation of oocytes in the Nili-Ravi buffalo. We hypothesized that good quality oocytes from Nili-Ravi buffalo would have diameter more than 120 um and the width of zona pellucida will be thinner in good quality oocytes as compared to poor quality oocytes. In the present study, the main features manifested were oocyte diameter, ooplasm diameter, width of zona pellucida, oocyte area and ooplasm area. The oocytes diameter was $149.76 \mu \mathrm{m}$ that is slightly higher than the previous study $(146.4 \mu \mathrm{m})$, more follicle size and oocyte diameter has a positive effect on the developmental competence in buffalo oocytes [Raghu et al., 2002]. In bovines the oocyte attains meiotic competence at 115 um while the competence to develop into a blastocyst is attained at the size of 120um [Martino et al., 1996]. There was the highest MII meiotic stage in bovine oocytes consisting of $>120 \mu \mathrm{m}$ oocyte diameter [Fair et al., 1995]. Similarly in canine the meiotic developmental competence was attained when oocyte diameter reached at $120 \mu \mathrm{m}$ [Oto $i$ et al., 2000].All these findings are in accordance with our present study. Thus, it is implied that oocytes having diameter more than 140um should be considered for further processing to achieve better developmental competence in Nili-Ravi buffalo.

The current study revealed that there was a strong correlation between oocyte diameter and ooplasm diameter in $\mathrm{A}+\mathrm{B}$ grade oocytes $(\mathrm{r}=0.7792)$ as compared to the $\mathrm{C}+\mathrm{D}$ grade oocytes $(\mathrm{r}=0.5967)$.

The present study demonstrated that there was a moderate correlation between oocyte diameter and the width of zona pellucida in $\mathrm{C}+\mathrm{D}$ grade oocytes $(\mathrm{r}=0.6530$ ) as compared to $\mathrm{A}+\mathrm{B}$ grade oocytes $(\mathrm{r}=0.5287)$. The result showed that the thickness of zona pellucida was more in poor quality oocytes as compared to good quality oocytes. These results are in accordance with the previous studies, higher thickness of zona pellucida has a negative effect on the developmental competence of oocytes in bovines [Hatif et al., 2016]. Similarly in humans, Zona Pellucida was more thicker in unfertilized oocytes $(18.9 \pm 4.0 \mu \mathrm{m})$ as compared to fertilized oocytes $(16.6 \pm 3.2 \mu \mathrm{m})$ [Bertrand et al., 1995]. There was a significant difference in the thickness of zona pellucida of unfertilized $(18.9 \mu \mathrm{m})$, fertilized $(16.4 \mu \mathrm{m})$ and polyspermic oocytes $(15.1 \mu \mathrm{m})$ in human [Bertrand et al., 1996]. Taken together, it is implied that the oocytes having more diameter and less thickness of zona pellucida should only be processed for better developmental competence in Nili-Ravi buffalo.

Conclusion: It was concluded that the greater the ooplasm diameter and lesser the width of zona pellucida give rise to the good quality oocytes in Nili-Ravi buffalo.
Ooplasm diameter in addition to oocyte diameter can be used as a marker for the categorization of good and poor quality oocytes. Therefore, it is implied that for better developmental competence the oocytes with bigger ooplasm and thinner zona pellucida should be processed.

Acknowledgements: This study was funded by NRPU project of Higher Education Commission (HEC), Islamabad, Pakistan. The authors are grateful to PAMCO (Punjab Agriculture \& Meat Company) for the provision of ovaries. We gratefully acknowledge Mr. Muhammad Wajahat Saleem, Research Associate at Embryology laboratory in the Central Laboratory Complex, UVAS, Ravi campus, Pattoki for providing constant help throughout the study.

\section{REFERENCES}

Bertrand, E., M. Van den Bergh, and Y. Englert (1995). Fertilization and early embryology: does zona pellucida thickness influence the fertilization rate?, Hum. Reprod. 10(5), 1189-1193.

Bertrand, E., M. Van den Bergh, and Y. Englert (1996). Clinical parameters influencing human zona pellucida thickness, Fertil. and steril. 66(3), 408411.

Fair, T., P. Hyttel, and T. Greve (1995). Bovine oocyte diameter in relation to maturational competence and transcriptional activity, Mol. Reprod. and Dev.42(4), 437-442.

Gabrielsen, A., P. R. Bhatnager, K. Petersen, and S. Lindenberg (2000). Influence of zona pellucida thickness of human embryos on clinical pregnancy outcome following in vitro fertilization treatment, J. Assist. Reprod. Genet. 17(6), 323-328.

Hatif, S. A., and N. Al Mustafa (2016). Influence of Zona Pellucida Thickness of Oocyte Maturation and In Vitro Fertilization in The Cow in Iraq.

Kastrop, P., M. Bevers, O. Destree, and T. A. Kruip (1990). Analysis of protein synthesis in morphologically classified bovine follicular oocytes before and after maturation in vitro, Mol. Reprod. and Dev. 26(3), 222-226.

Martino, A., N. Songsasen, and S. Leibo (1996), Development into blastocysts of bovine oocytes cryopreserved by ultra-rapid cooling, Biol. Reprod.54(5), 1059-1069.

Moore, S., and J. Hasler (2017). A 100-year review: Reproductive technologies in dairy science, J. Dairy Sci. 100(12), 10314-10331.

Mukaka, M. M. (2012), A guide to appropriate use of correlation coefficient in medical research, Malawi Med. J. 24(3), 69-71.

Neglia, G., B. Gasparrini, V. C. di Brienza, R. Di Palo, G. Campanile, G. A. Presicce, and L. Zicarelli 
(2003). Bovine and buffalo in vitro embryo production using oocytes derived from abattoir ovaries or collected by transvaginal follicle aspiration, Theriogenology, 59(5-6), 1123-1130.

Otoi, T., K. Yamamoto, N. Koyama, S. Tachikawa, and T. Suzuki (1997), Bovine oocyte diameter in relation to developmental competence, Theriogenology, 48(5), 769-774.

Otoi, T., M. Fujii, M. Tanaka, A. Ooka, and T. Suzuki (2000). Canine oocyte diameter in relation to meiotic competence and sperm penetration, Theriogenology, 54(4), 535-542.

Raghu, H., S. Nandi, and S. Reddy (2002). Follicle size and oocyte diameter in relation to developmental competence of buffalo oocytes in vitro, Reprod. Fertil. and Dev. 14(1), 55-61.

Vieira, L., C. Rodrigues, A. C. Netto, B. Guerreiro, C. Silveira, R. Moreira, M. Sá Filho, G. Bó, R. Mapletoft, and P. Baruselli (2014), Superstimulation prior to the ovum pick-up to improve in vitro embryo production in lactating and non-lactating Holstein cows, Theriogenology, 82(2), 318-324.

Yousaf, M. R., and K. R. Chohan (2003). Nuclear morphology, diameter and meiotic competence of buffalo oocytes relative to follicle size, Reprod. Fertil. and Dev. 15(4), 223-229. 